\title{
TOWARDS BETTER FINITE ELEMENT MODELLING OF ELASTIC RECOVERY IN SHEET METAL FORMING OF ADVANCED HIGH STRENGTH STEEL
}

\author{
Mohsen Safaei, Wim De Waele \\ Ghent University, Laboratory Soete, Belgium
}

\begin{abstract}
The first part of this study discusses the influence of element type on parameters such as accuracy of the FE simulation, simulation time and convergence. Guidelines on optimal implementation of element types are proposed. It is shown that an inappropriate choice of element type results in difficulties in convergence of the simulation or gives rise to problems such as shear locking in elements. In the second part of this study a series of finite element simulations using the Hill' 48 planar anisotropic yield criterion and a standard U-shape forming test based on the NUMISHEET'93 benchmark was performed. The effectiveness of different isotropic hardening laws and different contact models is investigated. The most appropriate hardening and contact definitions are defined from the viewpoint of optimal springback prediction. Finally, the influence of the orientation of sheet strips relative to the rolling direction on springback angles is evaluated.
\end{abstract}

Keywords Convergence, Element type, Springback, Hardening, Contact model

\section{INTRODUCTION}

Obtaining accurate results in finite element simulations demands an appropriate use of elements, contact and material definitions. It is important to know how their implementation affects computational expenses and the accuracy of the simulations. Regarding material definition for sheet metal forming processes, a yield function should address the mechanical anisotropy of the material to produce the true amount of yield stress in different directions with respect to the rolling direction. An appropriate hardening law relates the true stress to strain for different loading paths. A variety of hardening models have been employed in research such as simple isotropic hardening laws to the most advanced mixed isotropic-kinematic laws such as Yoshida-Uemori [1] and Teodosiu [2] which can model the elastic-plastic transition, stagnation of hardening and softening caused by reversed load path.

The first part of this paper reports on the influence of element type. Shell and solid elements are discussed, as is the order and integration of the elements. Some guidelines on element selection are listed in annex. The second part of this paper reports on the springback simulation of a U-shape forming test (NUMISHEET'93 benchmark). Different contact models and a variety of hardening laws are investigated.

Finally, the effect of sheet orientation with respect to the rolling direction on springback of an advanced high strength steel is evaluated.

\section{ELEMENT TYPE STUDY}

\subsection{Shell elements}

Simulations of sheet metal forming processes mostly utilize shell elements rather than solid elements. This is because shell elements can produce comparable accuracy as solid elements for less computational expense. One issue might be the change of shell element thickness during deformation. This thickness change can be calculated based on the simulated in-plane deformations: 


$$
\begin{gathered}
\varepsilon_{33}^{e l}=-\frac{v}{1-v}\left(\varepsilon_{11}^{e l}+\varepsilon_{22}^{e l}\right) \\
\varepsilon_{33}^{p l}=-\left(\varepsilon_{11}^{p l}+\varepsilon_{22}^{p l}\right) \\
\varepsilon_{33}=\varepsilon_{33}^{e l}+\varepsilon_{33}^{p l}
\end{gathered}
$$

A Poisson's ratio equal to zero will keep the shell thickness constant, which is suitable for small strain, large rotation analyses. For analysis of large strain applications such as sheet metal forming, the Poisson's ratio is set to 0.5 , which addresses the incompressibility of the material.

Either first-order or second-order elements can be employed. Second-order elements produce higher accuracy than first-order elements for "smooth" problems in which no complex contact conditions nor severe element distortions exist. This kind of elements can model complex geometrical curves with fewer elements and are very effective in bending dominated problems. Moreover, the second-order elements capture stress concentrations more effectively.

Generally, the Gaussian quadrature integration method is used to evaluate the material response at each integration point of an element. Using full versus reduced integration for an element might result in a significant change in simulation results.

Reduced integration uses a lower-order integration to form the element stiffness matrix, thus reducing running time especially in three dimensional simulations. More accurate results are generally expected when this lower integration method is applied for a second-order element. However, for first-order elements the application in which the element is used, defines the accuracy of the result regardless the order of integration used.
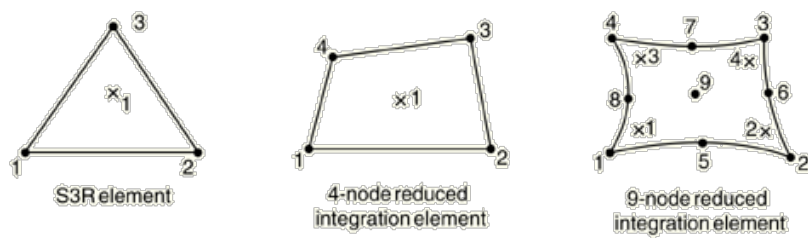

9-node reduced integration element

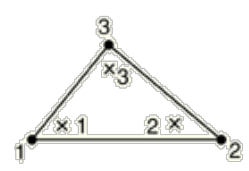

STRI3 element

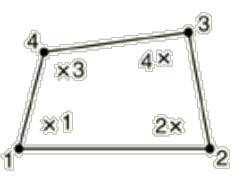

4-node full integration element
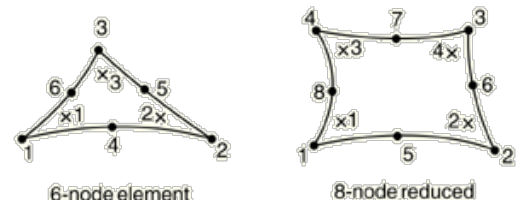

8-node reduced

integration element

Figure 1. Full-integration against reduced-integration for conventional shell elements [3]

\subsection{Hourglassing and shear locking}

Using solid elements for sheet metal forming simulations requires special care. For instance, inherently, first-order triangular and tetrahedral elements are overly stiff and exhibit slow convergence when increasing the number of elements, and therefore they are not suggested for stress analysis.

Shear locking and hourglassing are two major problems when using solid elements. The phenomenon of being overly stiff in bending is called shear locking. Fully integrated first order solid elements may suffer from this problem. Curved edges are expected for a beam element under pure bending moment. However, the linear geometry of a first order element does not allow bending in the element. Therefore it distorts by shear at the corners of the element (Figure 2), resulting in large shear stress in these regions. Erroneous displacement and stress may be observed because of shear locking. 


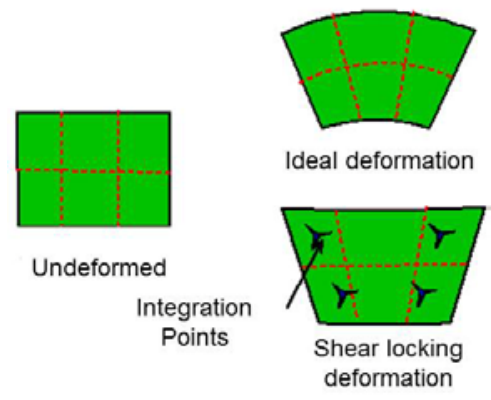

Figure 2. Shear locking for a first order fully integrated element

To avoid the shear locking problem, one may suggest using first order reduced integration elements. In this case, because there is no strain energy at the integration point, zero amounts of normal and shear stresses are obtained at this point. This produces an overly flexible structure, thus resulting in erroneous results such as wrong stress patterns. This problem is called hourglassing that can also be observed when only one layer of the second-order reduced-integration solid element is used. This can easily be solved by using two or more layers of elements. Less than one percent ratio of artificial energy to strain energy denotes no hourglassing problem in the simulation.

\subsection{General-purpose conventional shell elements}

The finite element software ABAQUS benefits from a database of general purpose shell elements (S3/S3R, S3RS, S4, S4R, S4RS, S4RSW, SAX1, SAX2, SAX2T, SC6R, and SC8R). These utilize the thick shell theory as the shell thickness increases and become thin shell elements as the thickness decreases.

\section{Using S4(R) elements}

The fully integrated general-purpose finite-membrane-strain shell element, S4, gives accurate solutions to in-plane bending problems, tolerates element distortion and avoids locking. Moreover, no hourglass control is needed for this element, neither for membrane nor bending mode. When compared to S4R reduced integration elements which possess only one integration point per element, the S4 elements with four integration points demand more computational time. In spite of higher computational time, the S4 element outperforms the S4R element type in areas where greater solution accuracy is required or for problems where in-plane bending is expected.

More detailed guidelines for element types are demonstrated in annex.

\section{SPRINGBACK SIMULATION OF AN ADVANCED HIGH STRENGTH STEEL}

This section discusses the finite element analysis of springback based on the NUMISHEET'93 U-shape forming test [4] for an advanced high strength steel (TRIP780). The geometry is shown in Figure 3; the sheet strips are $380 \mathrm{~mm}$ long by $35 \mathrm{~mm}$ wide by $0.81 \mathrm{~mm}$ thick. Only one quarter of the geometry was analysed due to the symmetrical physics of the model. FE simulations have been performed using ABAQUS v6.10. Special attention is paid to an appropriate representation of the material's stress-strain relation and contact definition. Figure 4 shows details about the definition of the springback angles $\theta_{1}$ and $\theta_{2}$. Figure 5 depicts positions of the parts before and after loading.

A mesh convergence study was performed and eventually a total of 1350 elements produced most accurate results. The blank was meshed with S4R elements (reduced integration general shell element) and the rigid parts (holder, punch and die) were meshed with S3D4 elements. Details of the employed contact model are presented in the following subsection. The anisotropy was addressed by using the Hill' 48 quadratic anisotropic equation [5]:

$\mathrm{F}\left(\sigma_{\mathrm{yy}}-\sigma_{\mathrm{zz}}\right)^{2}+\mathrm{G}\left(\sigma_{\mathrm{zz}}-\sigma_{\mathrm{xx}}\right)^{2}+\mathrm{H}\left(\sigma_{\mathrm{xx}}-\sigma_{\mathrm{yy}}\right)^{2}+2 \mathrm{~L} \tau_{\mathrm{xy}}{ }^{2}+2 \mathrm{M} \tau_{\mathrm{zx}}{ }^{2}+2 \mathrm{~N} \tau_{\mathrm{xy}}{ }^{2}=\mathrm{k}^{2}$

Where $\sigma_{x x}, \sigma_{y y}, \sigma_{z z}, \tau_{x y}, \tau_{z x}$ and $\tau_{x y}$ are the components of the Cauchy stress tensor defined in the orthotropic frame and $F, G, H, L, M$ and $N$ are the Hill's coefficients.

The material parameters used in simulations are summarized in Table 1.

Table 1 Material data for TRIP 780 AHSS steel

\begin{tabular}{lcccccc}
\hline Material & $\mathrm{E}(\mathrm{GPa})$ & $\mathrm{v}$ & Thickness $(\mathrm{mm})$ & $\mathrm{r}_{0}$ & $\mathrm{r}_{45}$ & $\mathrm{r}_{90}$ \\
\hline TRIP 780 & 200 & 0.3 & 0.81 & 1.02 & 0.95 & 1.14
\end{tabular}




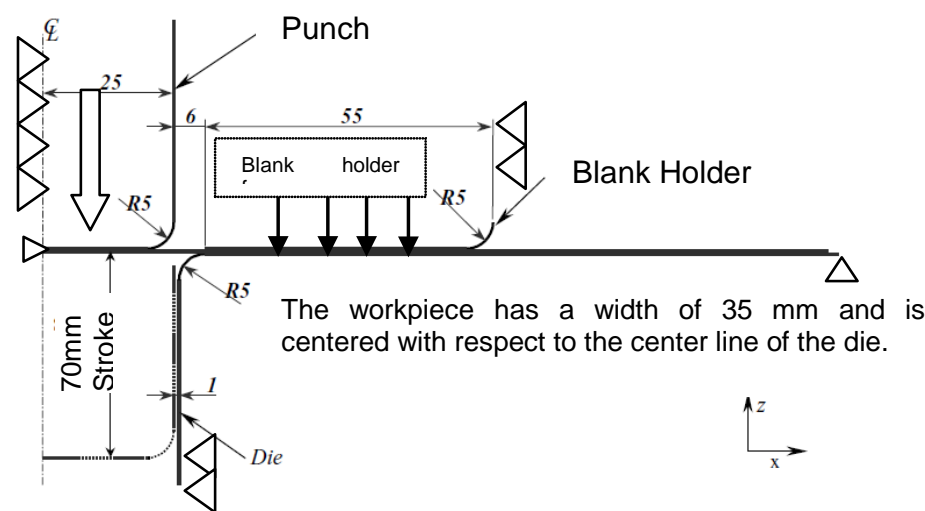

Figure 3. The NUMISHEET'93 benchmark geometry; (units: $\mathrm{mm}$ )
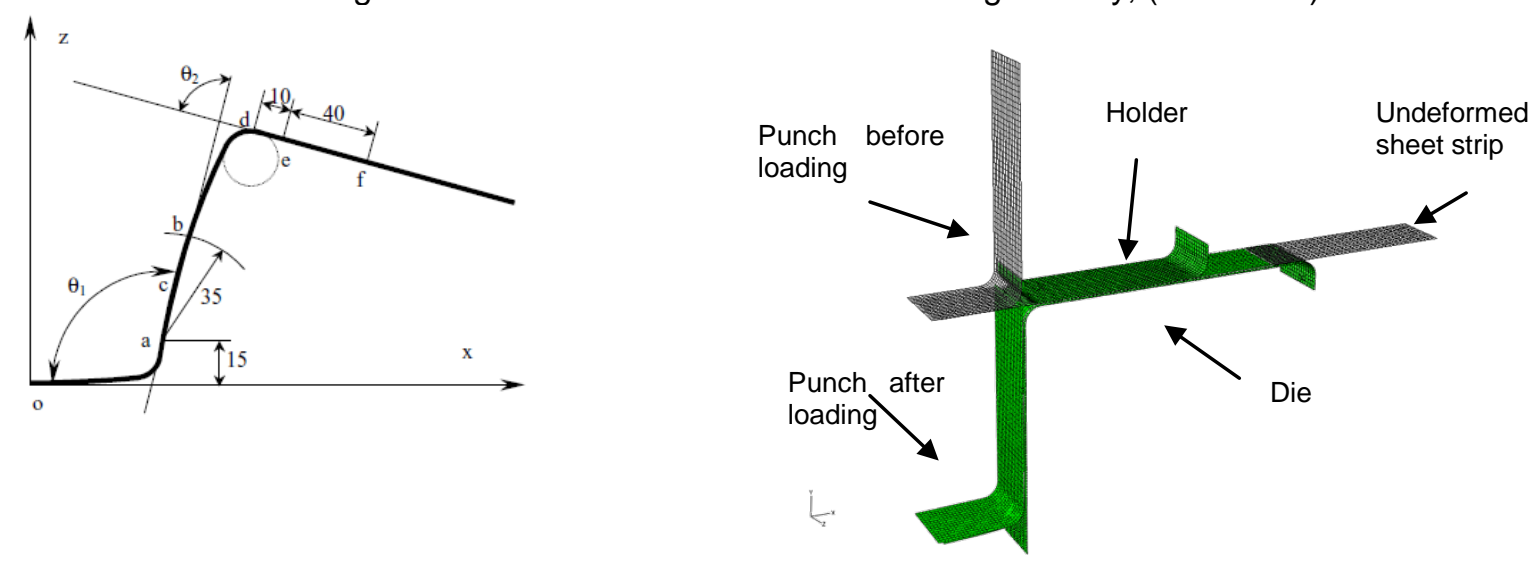

Figure 4. Variables defining springback; (units: $\mathrm{mm}$ ) [6]
Figure 5. Position of dies and sheet strip in FE model. Because of the symmetrical physics of the model, just one quarter of the model has been modelled.

\subsection{Contact models}

The effectiveness of three different contact models has been investigated. The first contact model used is the common Coulomb tangential friction with a friction coefficient of 0.165 . The second model is a linear penalty type of "hard" pressure-overclosure function combined with the tangential coulomb friction of 0.165. For this model, the contact pressure is linearly proportional to the normal penetration distance. A stiffness $K_{\text {lin }}$ of 0.01 was used. Finally, a softened (exponential) constraint enforcement method was used in which the surfaces begin to transmit contact pressure once the clearance between them reduces to $c_{0}$. The half of the sheet thickness and $5 \mathrm{MPa}$ were assigned for the parameters $c_{0}$ and $p_{0}$, respectively. In Abaqus/Standard the default $K_{\max }$ is 10 times the element stiffness. The functions of the two last contact models are demonstrated in Figure 6.
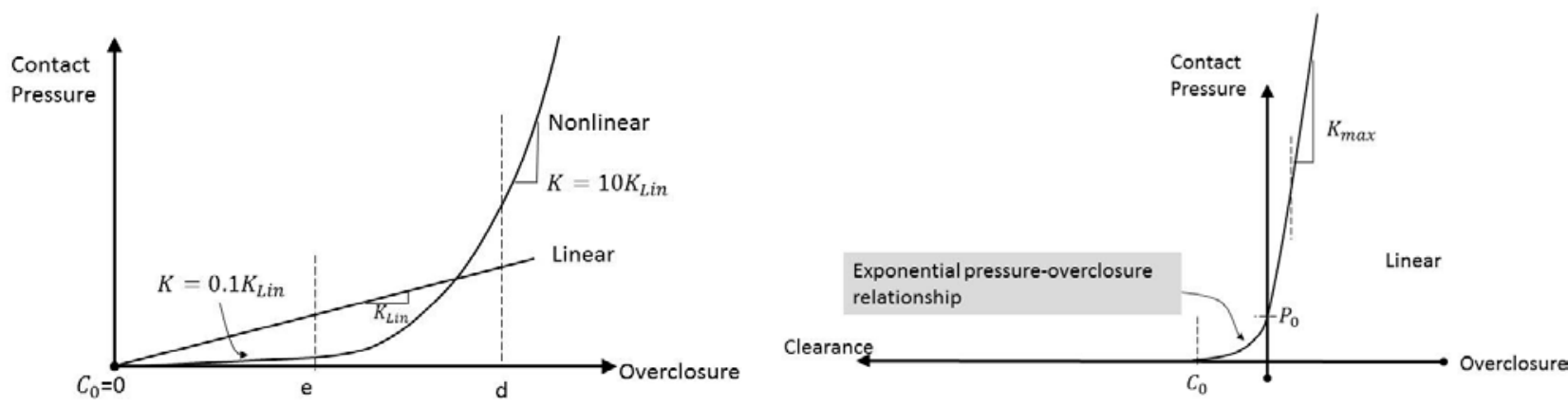

Figure 6. Linear and non-linear pressure-overclosure relation (left) and exponential (soft) contact pressureoverclosure relationship (right) [3]

Based on the performed simulations, it was concluded that the linear penalty contact model provides reasonable accuracy in less computational time. Therefore this model was selected for the study discussed in subsection 3.3. 


\subsection{Hardening laws}

To date, different material constitutive laws, from the simplest hardening laws and yield functions such as Ludwig and von Mises, respectively, up to the more accurate and complex mixed isotropic-kinematic hardenings such as Yoshida-Uemory [1], Teodosiu [2] and yield loci like Banabic [7] can be implemented in FE codes. In this study, different types of isotropic hardening laws are investigated and the most accurate hardening law (i.e. the best representation of stress-strain relation) is used in the springback prediction. Figure 7 presents a comparison between two simple isotropic hardening laws; Hollomon and Ludwig, with experimental data of TRIP 780. The Hollomon hardening model can be written as:

$\sigma=k \varepsilon^{n}$

and the Ludwig model:

$\sigma=\sigma_{0}+k \varepsilon^{n}$

where $K, \sigma_{0}$ and $n$ are material coefficients. The coefficients of the presented curves have been calculated by curve fitting using Newton's iteration method.

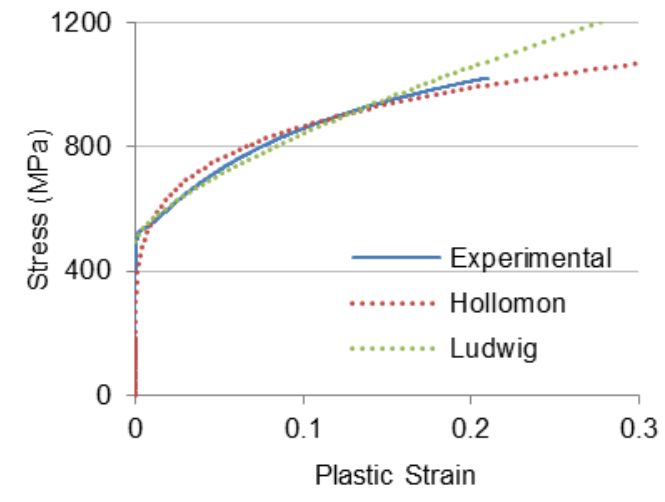

Figure 7.Comparison of Hollomon and Ludwig hardening laws with experimental stress-strain data of TRIP 780.

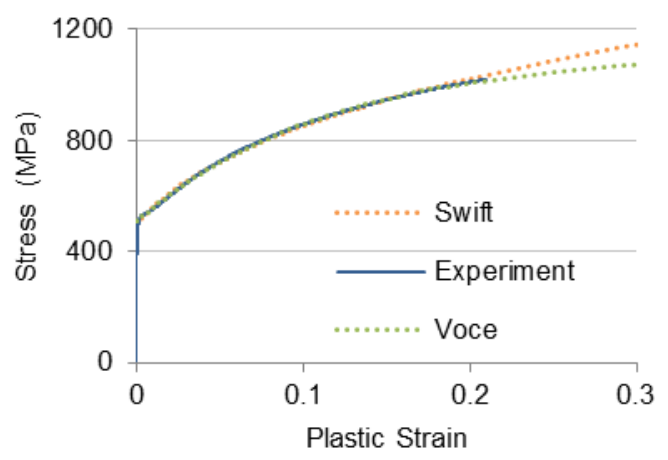

Figure 8.Comparison of Swift and Voce hardening laws with experimental stress-strain data of TRIP 780.

Apparently these two hardening laws are not accurate enough, neither before nor after UTS (end point of the experimental curve). For the region before UTS, the evaluation has been performed using asummation of least square errors between experimental stress-strain data and the fitted hardening curve. The evaluation for the region after UTS has been performed by comparing the tangents to the stress-strain curves at UTS.

Presented in Figure 8, the Swift and Voce hardening laws exhibit more accurate results than the previously evaluated simple hardening laws. The Swift and Voce laws can be written as Equation (7)and (8), respectively.

$\sigma=k\left(\varepsilon_{0}+\varepsilon\right)^{n}$

$\sigma=\sigma_{0,}+\sigma_{\text {sat }}\left(1-e^{-m \varepsilon}\right)$

Although agreeing better with experimental stress-strain data, more accuracy is desired especially after UTS. The conventional tensile test is not able to accurately predict the sheet behaviour after UTS because of non-uniform deformation occurring. In what follows, the possibility of a better extrapolation after UTS is investigated using a mixed Swift-Voce law

The combined Swift-Voce is written as:

$\sigma=(1-\alpha) k\left(\varepsilon_{0}+\varepsilon\right)^{n}+\alpha\left(\sigma_{0}+\sigma_{\text {sat }}\left(1-e^{-m \varepsilon}\right)\right)$

Figure 9 demonstrates that the combined Swift-Voce model fits perfectly with experimental data before and after uniform elongation. 


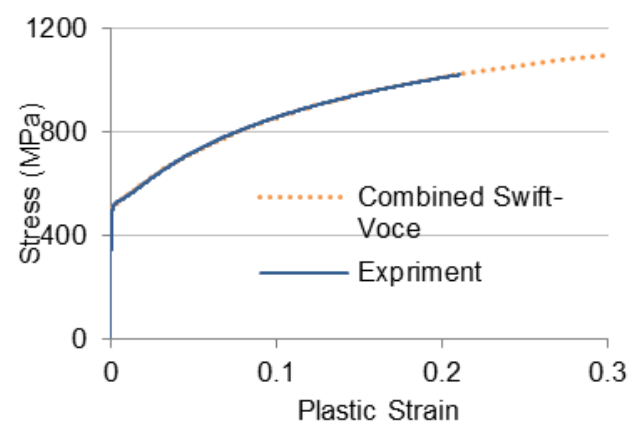

Figure 9. Combined Swift-Voce vs. experimental stress-strain curve.

\subsection{AHSS springback}

An ideal sheet metal product is desired to be without any elastic recovery. In the particular case of an Ubend shape, the walls are expected to be completely flat (i.e. $\theta_{1}=90^{\circ}=\theta_{2}$ ). In reality, the product underperforms the ideal expectations (Figure 10). An increase of $\theta_{1}$ and decrease of $\theta_{2}$, increases the springback. Changing the planar orientation of the sheet strip from 0 degrees to 45 degrees orientation with respect to the rolling direction, increases the springback (Figure 11). On the contrary, springback decreases when shifting from 45 degrees to 90 degrees orientation with respect to the rolling direction (Figure 11). The springback observed for the 90 degrees strip is similar to this of the 0 degrees strip.
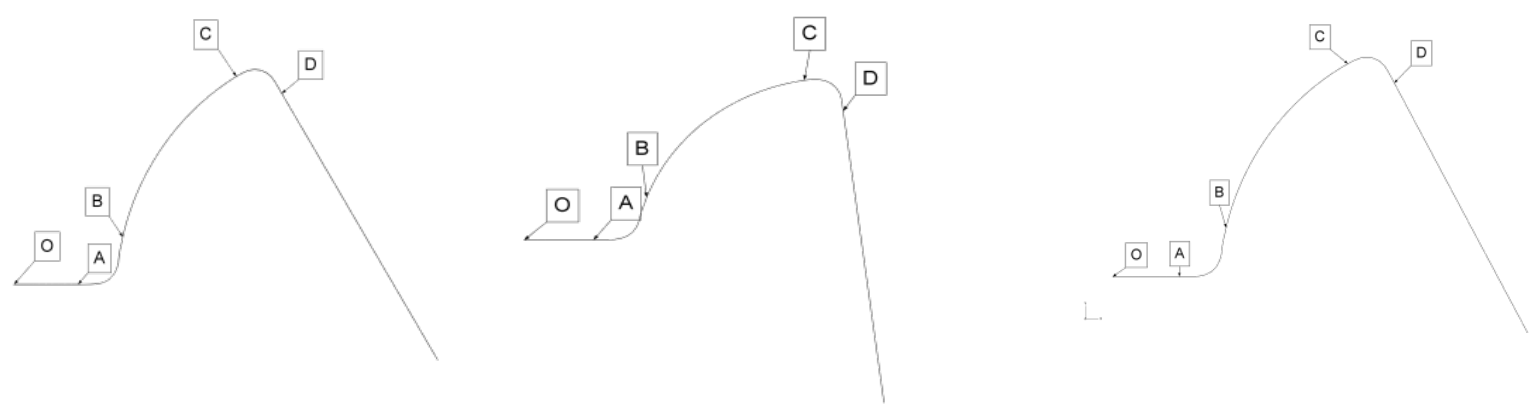

Figure 10. Illustration of springback after removal of the punch and holder for sheet strips with, from left to right, an orientation of 0,45 and 90 degrees with respect to the sheet's rolling direction. Note that only a half specimen is illustrated.

Figure 12 compares the thickness of the sheet strip at the last step of loading and after unloading for the various strip orientations. Although the $45^{\circ}$ orientation shows most elastic recovery, its thickness change in the vertical walls is limited. The $0^{\circ}$ and $90^{\circ}$ orientations exhibit comparable thinning of their vertical walls. 


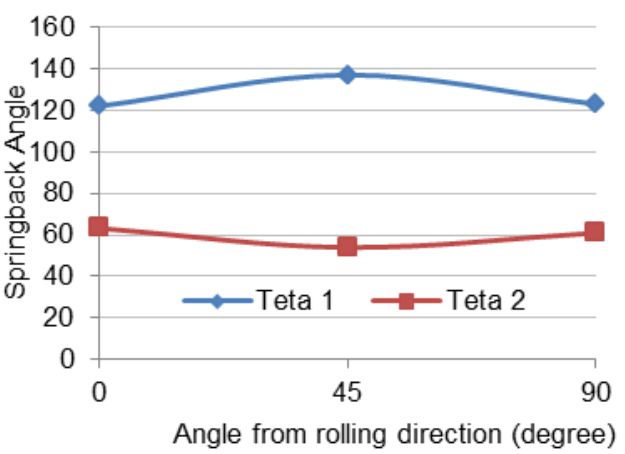

Figure 11. Springback angles vs. planar orientation

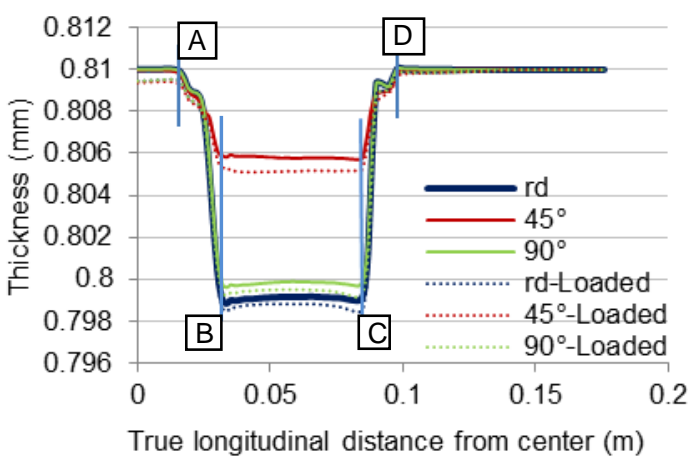

Figure 12. Comparison of sheet thickness before (dotted lines) and after unloading (full lines) for various sheet orientations. The letters refer to the positions indicated on Figure 10.

\section{CONCLUSIONS}

In the first part of this paper, appropriate employment of order and integration of elements are presented. Among three contacts model presented in the second part of the paper, it is found that the linear penalty type of "hard" pressure-overclosure function, applied together with a tangential coulomb friction of 0.165 produces enough accuracy and reduces the computational time interestingly. Moreover, the old Hill'48 Rfitted yield criterion was used to address the anisotropy of the material. Although isotropic hardening is only suggested for monotonic loading and not for reversed loading, it is used here as a case study. This is because the main goal was finding an stress-strain function that accurately fits experimental data before and after UTS. At the corners of a U-bend shape, reversed loading happens during forming. Isotropic hardening cannot model the Bauschinger effect by early re-yielding of material upon reversing the load. In case of severe strain path changes, and depending on the material, possibly severe change of hardening, hardening stagnation and softening will occur for which advanced hardening models such as YoshidaUemori [1] and Teodosiu [2] are suggested. These models will be used in future studies.

\section{NOMENCLATURE}

$\varepsilon_{\text {ii }} \quad$ Component of in-plane strain

$\varepsilon_{0} \quad$ Reference strain

$\sigma_{\mathrm{ij}} \quad$ Component of Cauchy stress tensor

$\sigma_{0} \quad$ Initial yield stress

$\sigma_{\text {sat }}$ Saturated amount of stress

v Poisson's ratio

E young's modulus

$\mathrm{r}_{\theta} \quad$ Lankford coefficient at $\theta^{\circ}$ with respect to the rolling direction

$K, n \quad$ Hardening parameters

$c_{0} \quad$ Parameter used for exponential pressure-overclosure contact relationship

\section{ACKNOWLEDGEMENTS}

The authors would like to acknowledge the financial support of the special research fund of Ghent University (BOF grant nr. 01J10608).

\section{REFERENCES}

1. Yoshida, F. and T. Uemori, A model of large-strain cyclic plasticity describing the Bauschinger effect and workhardening stagnation. International Journal of Plasticity, 2002. 18(5-6): p. 661-686. 
2. Haddadi, H. and S. Belhabib, An Attempt to Identify Hill's Yield Criterion Parameters Using Strain Field Measured by Dic Technique, in Experimental Analysis of Nano and Engineering Materials and Structures, E.E. Gdoutos, Editor. 2007, Springer Netherlands. p. 677-678.

3. Hibbitt Karlsson and Sorensen Inc, ABAQUS User's Manuals Version 6.10, Pawtucket, Rhode Island, USA.

4. NUMISHEET'93. in Proceedings of the second international conference of numerical simulation of 3-D sheet metal forming processes. 1993. Isehara, Japan.

5. Hill, R., The mathematical theory of plasticity. The Oxford engineering science series. 1950, Oxford,: Clarendon Press. ix, 356 p.

6. Gomes, C., O. Onipede, and M. Lovell, Investigation of springback in high strength anisotropic steels. Journal of Materials Processing Technology, 2005. 159(1): p. 91-98.

7. Banabic, D., et al., Increasing the robustness of the sheet metal forming simulation by the prediction of the forming limit band. NUMIFORM '07: Materials Processing and Design: Modeling, Simulation and Applications, Pts I and II, 2007. 908: p. 171-176. 


\section{ANNEX}

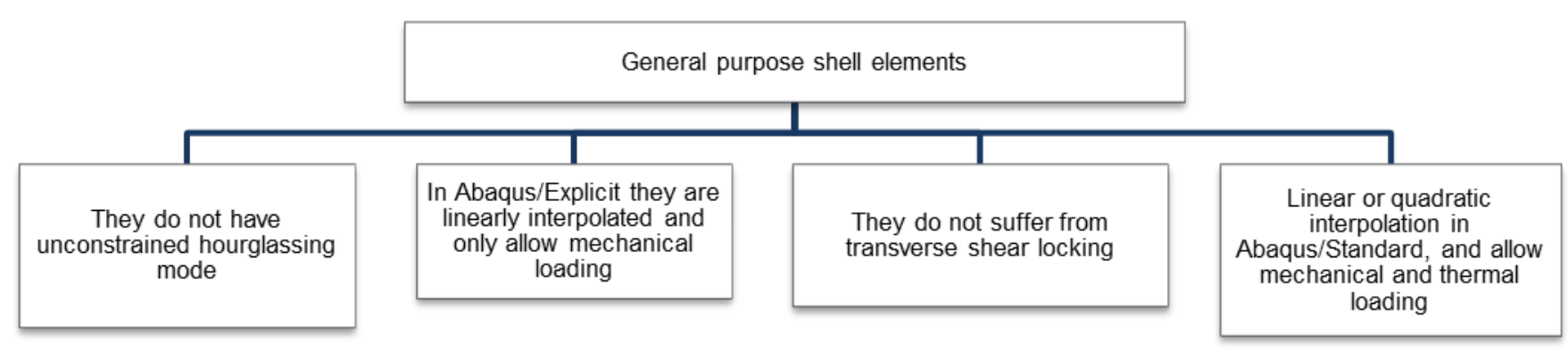

Figure 13. Some remarks on general purpose shell elements

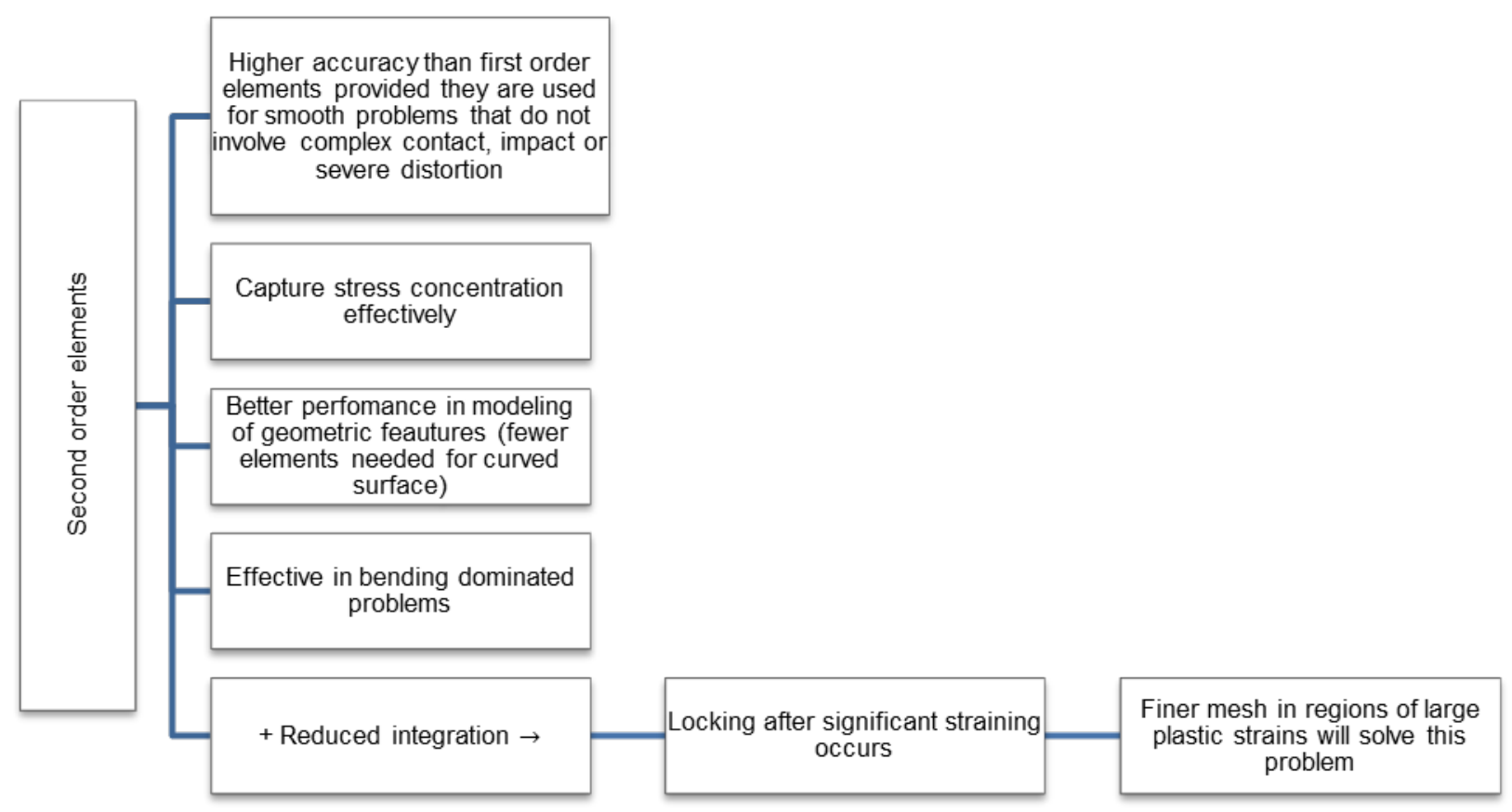

Figure 14. Guidelines for using second order elements 


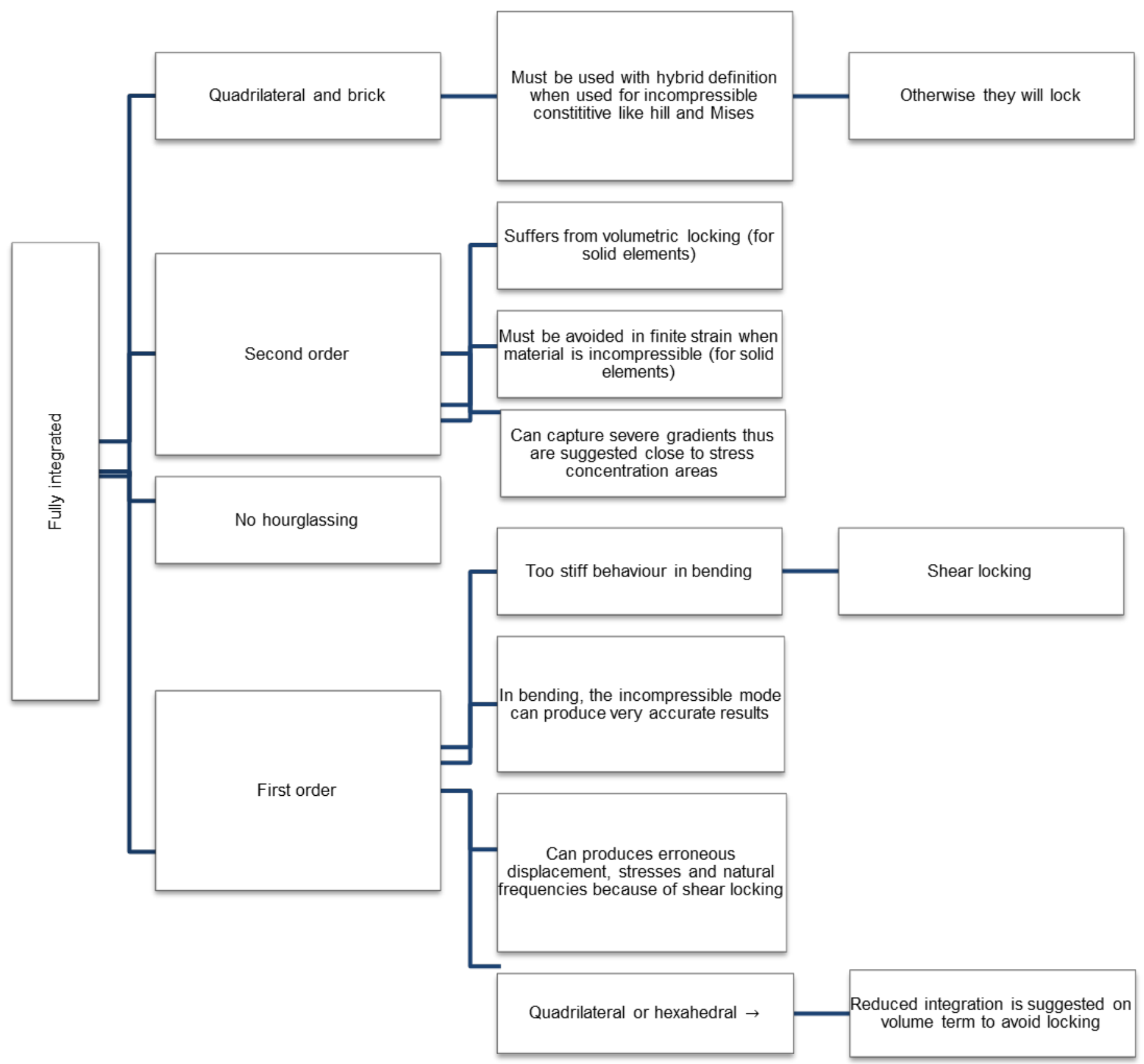

Figure 15. Guidelines for using fully integrated elements 


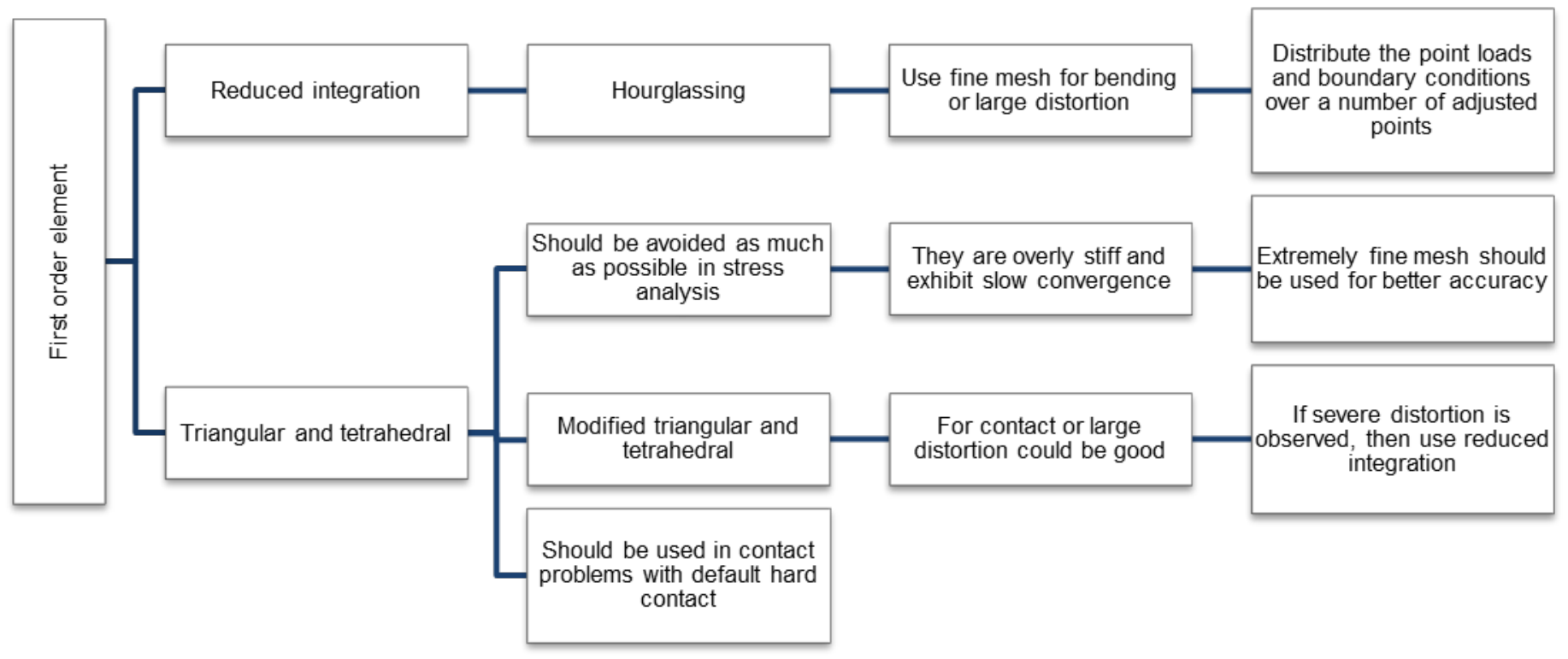

Figure 16. Some remarks on first order shell elements 